\title{
UNIQUE CONTINUATION THEOREMS FOR SOLUTIONS OF PARTIAL DIFFERENTIAL EQUATIONS
}

\author{
BY M. S. BAOUENDI AND E. C. ZACHMANOGLOU ${ }^{1}$ \\ Communicated by E. M. Stein, March 29, 1977
}

In this note we present a new general unique continuation theorem for solutions of linear P.D.E.'s with analytic coefficients. The solutions are assumed to vanish of infinite order on manifolds of codimension $\geqslant 1$. Holmgren's theorem and the unique continuation property of solutions of elliptic equations are particular cases of this theorem. Some new unique continuation results are also given for certain hyperbolic equations (and inequalities) with nonanalytic coefficients.

Let $\Omega$ be an open set of $\mathbf{R}^{n}$ and $P(x, D)$ be a linear partial differential operator of order $m$, with analytic coefficients in $\Omega$. We denote by $p_{m}$ the principal symbol of $P$ and by $\Sigma$ the characteristic set of $P$ contained in $T^{*}(\Omega) \backslash 0$, i.e.

$$
\sum=\left\{(x, \xi) ; x \in \Omega, \xi \in \mathbf{R}^{n} \backslash\{0\}, p_{m}(x, \xi)=0\right\}
$$

If $M$ and $N$ are two differentiable manifolds contained in $\Omega, M \subset N$, and if $u$ is a continuous function defined in $N$, we say that $u$ vanishes of infinite order on $M$ if, for all $\alpha \in \mathbf{R}$, the function $x \rightarrow d(x, M)^{\alpha} u(x)$ is bounded in any compact set of $N$. Here $d(x, M)$ denotes the distance of $x$ from $M$.

We say that the manifold $M$ is P-noncharacteristic if the normal bundle of $M$ (in $T^{*}(\Omega) \backslash 0$ ) does not intersect $\Sigma$, i.e., for all $x \in M$ and $\xi \in \mathbf{R}^{n} \backslash\{0\}$ normal to $M$ at $x, p_{m}(x, \xi) \neq 0$. We have:

TheOREM 1. Let $M$ and $N$ be two analytic manifolds in $\Omega, M \subset N$, and assume that $M$ is $P$-noncharacteristic. There is a neighborhood $V$ of $M$ in $N$, such that if $u$ is a continuous function in $\Omega$ satisfying: (i) $P u=0$ in $\Omega$, and (ii) the restriction of $u$ to $N$ vanishes of infinite order on $M$, then $u$ must vanish in $V$.

Note that $N$ can be taken to be $\Omega$. Condition (ii) may be replaced by a weaker condition; for example, if $M$ divides $N$ into two sides, then it is enough

AMS (MOS) subject classifications (1970). Primary 35A05, 35A20, 35A30; Secondary $35 \mathrm{~L} 10,35 \mathrm{~L} 60,35 \mathrm{R} 05$.

Key words and phrases. Uniqueness, unique continuation, P.D.E.'s with analy tic coefficients, noncharacteristic manifolds, hyperbolic equations and inequalities, singular coefficients.

1 Research supported by the National Science Foundation, Grant no. MCS 7510361A1 for the first author and Grant No. MCS 76-06442 for the second author. 
to assume that the restriction of $u$ to $N$ vanishes of infinite order on $M$ from one side only.

IdeA of Proof of Theorem 1. If in addition $u \in C^{m}(\Omega)$ the theorem may be proved using the result of F. John [4] which asserts the analyticity with respect to $t$ of integrals of the form

$$
\int_{M_{t}} u g d s
$$

where $g$ is analytic and $M_{t}$ are certain analytic deformations of $M$ in $N$.

For continuous $u$, we use the known result about analytic wave front sets of solutions of $P u=0$ (see [3], [5]). By an appropriate singular change of variables, we reduce the problem to the case where $u$ vanishes outside a set whose projection on $M$ is compact, and then we use the result of Hormander [3] concerning the analyticity of certain integrals of form (1).

It is well known from examples of Cohen and Plis that Theorem 1 cannot be generally true if the coefficients of $P$ are assumed to be $C^{\infty}$ (even when $N=$ $\Omega$ and $M$ is a hyperplane). However it is known that, for second order elliptic equations with nonanalytic coefficients, Theorem 1 holds with $N=\Omega$ and $M$ a point in $\Omega$. (See for example [2] and its references.)

For hyperbolic (or ultrahyperbolic) second order equations with nonanalytic coefficients we can obtain unique continuation results from noncharacteristic manifolds. Here we restrict ourselves to equations with principal part the wave operator $\Delta_{x}-\partial_{t}^{2}$ (with $\Delta_{x}=\Sigma_{1}^{n} \partial_{x_{i}}^{2}$ ) and the manifold $M$ being a line segment of the $t$-axis. Actually the method of proof allows us to state our results for inequalities.

For $x \in \mathbf{R}^{n}$ we set $r=\left(\sum_{i=1}^{n} x_{i}^{2}\right)^{1 / 2}$ and $x=r \theta$ where $\theta$ varies over the unit sphere $S_{n-1}$. For $R>0$, let

$$
D_{R}=\left\{(x, t) \in \mathbf{R}^{n+1} ; r+|t|<R\right\} .
$$

Theorem 2. Let $u \in C^{2}\left(D_{R}\right)$ and assume that there exist positive constants $C_{1}$ and $C_{2}$ such that

$$
\begin{gathered}
\left|\Delta_{x} u-\partial_{t}^{2} u\right| \leqslant C_{1}\left(r^{-1}|\operatorname{grad} u|+r^{-2}|u|\right) \quad \text { in } D_{R}, \text { and } \\
\int_{S_{n-1}}\left|\operatorname{grad}_{\theta} u(r \theta, t)\right|^{2} d \theta \leqslant C_{2} \int_{S_{n-1}}|u(r \theta, t)|^{2} d \theta
\end{gathered}
$$

for $r+|t|<R$. If $u$ and $\operatorname{grad} u$ vanish of infinite order on the line segment $\{x=0,|t|<R\}$ then $u$ must vanish in $D_{R}$.

We have been unable to prove Theorem 2 without condition (3). It should be noted that the class of functions satisfying condition (3) includes: (a) Functions which have finite expansions with respect to an orthonormal basis of spherical harmonics in $L^{2}\left(S_{n-1}\right)$; in particular, functions which depend only on 
$r$ and $t$. (b) Functions of the form $u(x, t)=h(r, t) v(r \theta, t)$ where $h \in C^{2}$ for $r+|t| \leqslant R$ and $v$ is analytic in $r$ and $t$ for $r+|t| \leqslant R$.

Inequality (2) is satisfied for example by solutions of the nonlinear equation

$$
\Delta_{x} u-\partial_{t}^{2} u=F(x, t, u, \operatorname{grad} u)
$$

provided that

$$
|F(x, t, u, \operatorname{grad} u)| \leqslant C_{3}\left(r^{-1}|\operatorname{grad} u|+r^{-2}|u|\right)
$$

in $D_{R}$, for some positive constant $C_{3}$. In particular, inequality (2) is satisfied by solutions of the linear equation

$$
\Delta_{x} u-\partial_{t}^{2} u+\frac{1}{r} L\left(x, t, D_{x}, D_{t}\right) u+\frac{c(x, t)}{r^{2}} u=0
$$

where $L$ is a first order linear differential operator, the coefficients of $L$ and $c$ being bounded functions in $D_{R}$.

When the lower order terms in (4) depend only on $r$ and $t$ condition (3) can be dropped. We have

THEOREM 3. Let $u \in C^{2}\left(D_{R}\right)$ and satisfy

$$
\Delta_{x} u-\partial_{t}^{2} u+\frac{a(r, t)}{r} \partial_{r} u+\frac{b(r, t)}{r} \partial_{t} u+\frac{c(r, t)}{r^{2}} u=0
$$

in $D_{R}$, where $a, b, c$ are bounded functions for $r+|t|<R$. If $u$ and $\operatorname{grad} u$ vanish of infinite order on the line segment $\{x=0,|t|<0\}$, then $u$ must vanish in $D_{R}$.

IDEA of Proof of Theorems 2 AND 3. Theorem 2 is a consequence of the following weighted $L^{2}$ inequality:

For every $u \in C^{2}\left(\bar{D}_{R}\right)$ satisfying condition (3) and such that $u$ and its first and second order derivatives vanish of infinite order on $\{x=0,|t| \leqslant R\}$, there is $C>0$ such that, for all sufficiently large $\gamma$,

$$
\int_{D_{R}}\left(r^{-2}|\operatorname{grad} u|^{2}+r^{-4}|u|^{2}\right) r^{-\gamma} d x d t \leqslant \frac{C}{\gamma} \int_{D_{R}}\left|\Delta_{x} u-\partial_{t}^{2} u\right|^{2} r^{-\gamma} d x d t .
$$

This inequality is proved by the method of multipliers applied to equations with coefficients which are singular in $r$.

Theorem 3 is proved by expanding $u$ in spherical harmonics and applying Theorem 2 to each component. Note that each component satisfies a singular hyperbolic equation of the type for which uniqueness has also been proved in $[1]$.

\section{REFERENCES}

1. S. Alinhac, Problémes de Cauchy pour des opérateurs singuliers, Bull. Soc. Math. France 102 (1974), 280-315. 
2. H. Cordes, Über die eindeutige Bestimmtheit der Lösungen elliptischer Differen. tialgleichungen durch Anfangsvorgaben, Nachr. Akad. Wiss. Göttingen Ila (1956), 239-258. MR 19, 148.

3. L. Hörmander, Uniqueness theorems and wave front sets for solutions of linear differential equations with analytic coefficients, Comm. Pure Appl. Math 24 (1971), 671704. MR $45 \# 3917$.

4. F. John, On linear partial differential equations with analytic coefficients, Comm. Pure Appl. Math. 2 (1949), 209-253. MR 12, 185.

5. M. Sato, T. Kawai and M. Kashiwara, Microfunctions and pseudo-differential equa. tions, Lecture Notes in Math., no. 287, Springer-Verlag, Berlin and New York, 1973, pp. $265-529$.

DEPARTMENT OF MATHEMATICS, PURDUE UNIVERSITY, WEST LAFAYETTE, INDIANA 47907 